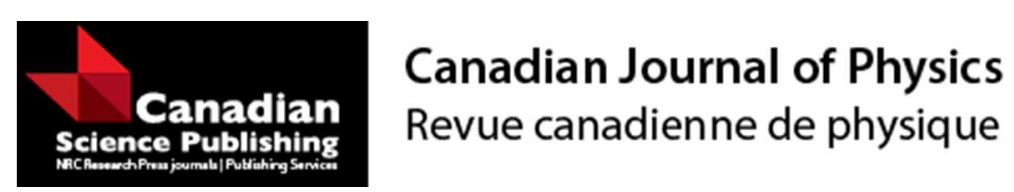

\title{
Householder methods for quantum circuit design
}

\begin{tabular}{|r|l|}
\hline Journal: & Canadian Journal of Physics \\
\hline Manuscript ID & cjp-2015-0490.R1 \\
\hline Manuscript Type: & Article \\
\hline Date Submitted by the Author: & $25-$ Sep-2015 \\
\hline Complete List of Authors: & $\begin{array}{l}\text { Urias, Jesus; Universidad Autonoma de San Luis Potosi, Instituto de Fisica } \\
\text { Quiñones, Diego; University of Leeds }\end{array}$ \\
\hline Keyword: & $\begin{array}{l}\text { Householder factorizations in tensor-product spaces, Algorithmic synthesis } \\
\text { of quantum circuits, Quantum simulators, Quantum-gate resolution of } \\
\text { unitary operations, BInary Gray codes }\end{array}$ \\
\hline & \\
\hline
\end{tabular}




\title{
HOUSEHOLDER METHODS FOR QUANTUM CIRCUIT DESIGN
}

\author{
Jesús Urías $^{a}$ and Diego A. Quiñones ${ }^{b}$ \\ a Instituto de Física, UASLP. San Luis Potosí, SLP, México. \\ b University of Leeds, West Yorkshire, UK
}

\begin{abstract}
Algorithms to resolve multiple-qubit unitary transformations into a sequence of simple operations on one-qubit subsystems are central to the methods of quantum-circuit simulators. We adapt Householder's theorem to the tensor-product character of multi-qubit state vectors and translate it to a combinatorial procedure to assemble cascades of quantum gates that recreate any unitary operation $U$ acting on $n$-qubit systems. $U$ may be recreated by any cascade from a set of combinatorial options that, in number, are not lesser than super-factorial of $2^{n}, \prod_{j=1}^{2^{n}}(j !)$. Cascades are assembled with one-qubit controlled-gates of a single type. We complement the assembly procedure with a new algorithm to generate Gray codes that reduce the combinatorial options to cascades with the least number of CNOT gates. The combined procedure - factorization, gate assembling and Gray ordering - is illustrated on an array of three qubits.
\end{abstract}

\footnotetext{
aurias@ifisica.uaslp.mx

b pydaqo@leeds.ac.uk
}

PACS Nos: 03.67. Ac 03.65.Fd 


\section{INTRODUCTION}

The large number of applications [1] and the steep technological advancement in the coherent control of quantum systems [2] constitute a promise that quantum simulators will become a valuable practical research tool. Important design tools for quantumcircuit simulators [3] (based on the evolution of multi-qubit systems) are the algorithms for factoring the overall evolution operator into quantum operations acting on smaller subsystems. For finite-dimensional systems, standard methods for matrix factorizations constitute a frequent approach [4].

In this article we develop a factorization method strictly based upon the tensor-product character of the state space of multi-qubit systems that we apply to provide a rigorous methodology to assemble a combinatorially reach collection of quantum circuits that recreate any given unitary operation. Our interest was focused on the development of a procedure that generates mathematically exact maps of the tensor-product factors of a unitary operation into quantum gates of a single type. Finding a theoretically feasible Hamiltonian that approximates the desired unitary transformation and then finding among all possible factorizations an efficient reconstruction (with polynomial resources) in terms of a standardized class of quantum gates are two difficult problems that we do not address [6].

The mathematical background of our methodology is a very well known theorem by Householder, which provides methods to generate exact resolutions of unitary transformations into simpler factors [7]. The Householder approach has previously been applied to assemble experimental realizations of finite unitary operators [8, 9]. However, for the interests of quantum simulators, a serious limitation of the previous physical realizations of Householder factorizations is that they were developed for situations where the elements of $\mathrm{U}(\mathrm{N})$ are propagators in the state space of a single quantum object. Such realizations lose the richness of the tensor product structure that the state space of any multi-particle system has [3]. In reference [9] the Householder factors in the representation of an element of $\mathrm{U}(N)$ gradually "push forward" a single quNit — a quantum object with $N$ states. Similarly, the realization of an element of $\mathrm{U}(N)$ in [8] concerns a single photon in the $N$ spatially-differentiated states that are provided by a linear optical setup. Such 
single-particle implementations of Householder reflections are of no use for multi-particle systems. Even in the case of multi-qubit systems the matrix factorization methods that are applied [4] do not exploit the tensor-product character of state vectors.

Our aim is to adapt the mathematical robustness and flexibility of Householder's theorem to the tensor-product character of multi-qubit states and then translate it into methods to assemble unitary operations on $n$-qubit states as cascades of quantum gates. The necessary background for our multi-qubit implementations of Householder factorizations is presented in Section 2. The mathematical task is to transform an input orthonormal vector basis $\left(x_{i}\right)_{i=0}^{N-1}$ into the target basis $\left(y_{i}\right)_{i=0}^{N-1}$, orthonormal too. The first step to attain it is Lemma 2.1, where we explain how to prepare successions of Householder reflections to transform a given vector $y$ into another vector $x$, sequentially. Under the conditions assumed by Lemma 2.1, we extract from its proof a recursion rule to generate sequences of reflections that connect a given pair of vectors. We let the term "block of reflections" denote the products of reflections that are provided by Lemma 2.1. The main result of Section 2 is Proposition 2.1, where we establish conditions to generate sequences of blocks of reflections that reproduce a given unitary transformation $U:\left(x_{i} \mapsto y_{i}\right)_{i=0}^{N-1}$. The special case of Householder factorizations in the form of $\mathrm{U}(2)$ blocks is our basis to implement unitary actions on $n$-qubit systems as cascades of controlled one-qubit "rotations". Then, the parameterization of the $\mathrm{U}(2)$ elements in a block are worked out in full detail.

In Section 3, the mathematical background that was presented in Section 2 is adapted to the product structure of the vector space $\otimes^{n} \mathbb{C}^{2}$, which is the state space of a system of $n$ qubits. The hypotheses of Proposition 2.1 are reformulated in Section 3 in the form of a factoring scheme for the elements of $\mathrm{U}\left(2^{n}\right)$ when they are represented in the standard product basis for the $n$-qubit states. A very rich combinatory of Householder factorizations that is inherent to the product character of multi-qubit states puts the lower bound for the number of $\mathrm{U}(2)$ factorizations of a given operation in super-factorial of $2^{n}$, which is $\prod_{j=1}^{2^{n}} j$ !. This vast set of combinatorial options to resolve a unitary action on $n$-qubit systems into simpler factors has been disregarded in previous factorization schemes [5] and is not available for single-particle $(n=1)$ implementations. 
The mathematics of factorizations are translated to the language of quantum gates in Section 4. Therein, every $\mathrm{U}(2)$ reflection in a block is given the form of a standardized controlled quantum gate acting on a single qubit line. We call it a Householder gate (or H-gate for short). All the elements upon which H-gates are constructed are given the form of Lemma 4.1.

All the partial results presented so far coalesce to form our main result, which consists of a collection of combinatorial methods to assemble arrays of H-gates to recreate any unitary operation on a system of $n$ qubits. This result is Theorem 5.1 in Section 5 . The hard direction of the proof incorporates the results proved in Proposition 2.1 and Lemma 4.1 as a combinatorial procedure for the systematic production of all the cascades of H-gates that — within the vast set of Householder factorizations available for multiqubit systems - recreate a given unitary transformation. By the end of Section 5 we have converted Householder's theorem into a combinatorial procedure carried out on the binary words that encode the tensor-product character of multi-qubit states.

In Section 6 we complement the assembly method presented in Section 5 with a new algorithm to generate Gray codes $[11,12]$ - lists of binary words in a matrix formappropriate to minimize the number of CNOT gates in a cascade of H-gates. We did not address the problem of minimizing the number of H-gates in a cascade.

The combined procedure - factorization and Gray ordering - is applied to assemble, step by step, a cascade of H-gates that reproduces a generic unitary action on an array of three qubits.

\section{Time evolution as a succession of Reflections}

Let $U$ be a unitary time-step evolution in the vector space $V$ that takes the orthonormal basis of vector states $\left(x_{i}\right)_{i=0}^{N-1}$ into the vector basis $\left(y_{i}\right)_{i=0}^{N-1}$, orthonormal too. The evolution step $U$ takes $x_{i}$ into $y_{i}$. The tensor product structure of $n$-qubit states is our target but for the subject of the present section is unnecessary. We defer until next section the tensor product structure of $n$-qubits states.

We know that a transformation $U$ is unitary if, and only if, it is realizable as a succession of Householder reflections [7]. The convenience of reflections when conceiving a physical 
implementation of $U$, e.g. as a quantum circuit, is their ability to exchange two given vectors, $x$ and $y$, without touching the subspace that is orthogonal to them.

Considering vectors $x$ and $y$ as the input data, they determine the reflection vector $r=r(x, y)=z\|x\| y-\|y\| x$, with complex phase factor $z=z(x, y)=y^{*} x /\left|y^{*} x\right|$ (the interior product is being denoted by $y^{*} x$ ). Vector $r$ is the only direction to be reversed by the Householder reflection

$$
\mathrm{h}_{x, y} u:= \begin{cases}-u, & u \in \operatorname{span}(r) \\ u, & u \in \operatorname{span}(r)^{\perp} .\end{cases}
$$

in order to produce, up to a phase factor, the required exchange

$$
y \leftrightarrow \mathrm{h}_{x, y} y=\left(z^{*}\|y\| /\|x\|\right) x
$$

The one-dimensional subspace that vector $r$ spans is denoted by $\operatorname{span}(r)$ and its orthogonal complement is denoted by $\operatorname{span}(r)^{\perp}$. The parameterization of the Householder reflection $\mathrm{h}_{x, y}$ by the reflection vector $r(x, y)$ fits nicely for our interests.

The one-step exchange in (2) is the base case of a multi-step exchange of $x$ and $y$, $y \leftrightarrow q_{1} \leftrightarrow \cdots \leftrightarrow q_{s} \leftrightarrow x$, going through a number $s$ of intermediate vectors $q_{1}, \ldots, q_{s}$. Every pair $\left(x, q_{i}\right)$ will determine a reflection vector $r\left(x, q_{i}\right)$ and we will be looking for the simplest intermediate exchange actions.

Lemma 2.1. Let $x, y \in \mathbb{C}^{N} \equiv V$, normalized to $\|x\|=\|y\|=1$. For a positive $s<N$ let $V=\operatorname{span}(x) \oplus T_{1} \oplus \cdots \oplus T_{s}$ be an orthogonal direct sum decomposition of $V$. Then, for every $i=1, \ldots, s$, there exists a vector $q_{i} \in \operatorname{span}(x) \oplus T_{i}$ such that

$$
x=z \mathrm{~h}_{x, q_{s}} \cdots \mathrm{h}_{x, q_{2}} \mathrm{~h}_{x, q_{1}} y
$$

where $z$ is a phase factor.

Proof. Proceeds by induction in $s$ and for an arbitrary $\operatorname{dim}(V)=N>2$. The base case $(s=1)$ consists of the vector $q_{1}=y$ and the single reflection in (2). Then, we prove (3) holds for $s+1<N$, with $V=\operatorname{span}(x) \oplus T_{1} \oplus \cdots \oplus T_{s} \oplus T_{s+1}$, under the assumption that it holds for $s>0$. Let $W_{s}:=T_{s} \oplus T_{s+1}$. By assumption

$$
x=z \mathrm{~h}_{x, \bar{q}_{s}} \mathrm{~h}_{x, q_{s-1}} \cdots \mathrm{h}_{x, q_{1}} y,
$$


with $\bar{q}_{s} \in \operatorname{span}(x) \oplus W_{s}=\operatorname{span}(x) \oplus T_{s} \oplus T_{s+1}$. Undoing the last reflection $\mathrm{h}_{x, \bar{q}_{s}}$ in (4) we have that

$$
\mathrm{h}_{x, q_{s-1}} \cdots \mathrm{h}_{x, q_{2}} \mathrm{~h}_{x, q_{1}} y=z^{*} \mathrm{~h}_{x, \bar{q}_{s}} x=: \bar{y}_{s-1}=: q_{s}+q_{s+1}^{\prime}
$$

where $q_{s} \in \operatorname{span}(x) \oplus T_{s}$ and $q_{s+1}^{\prime} \in T_{s+1}$ constitute a direct sum decomposition of $\bar{y}_{s-1}$. By applying $\mathrm{h}_{x, q_{s}}$ we get

$$
\mathrm{h}_{x, q_{s}} \mathrm{~h}_{x, q_{s-1}} \cdots \mathrm{h}_{x, q_{2}} \mathrm{~h}_{x, q_{1}} y=: \bar{y}_{s}=z_{s}^{*}\left\|q_{s}\right\| x+q_{s+1}^{\prime} .
$$

The proof concludes by applying the reflection $\mathrm{h}_{x, q_{s+1}}$, with $q_{s+1}:=z_{s}^{*}\left\|q_{s}\right\| x+q_{s+1}^{\prime}$, and noticing that unitarity implies that $\left\|q_{s+1}\right\|=1$.

The sequence of vectors $\left(q_{i}\right)_{i=1}^{s}$ connecting the $x-y$ exchange in (3) are determined by the decomposition $V=\operatorname{span}(x) \oplus T_{1} \oplus \cdots \oplus T_{s}$ that is adopted in the hypotheses of Lemma 2.1. The intermediate vectors $\left(q_{i}\right)_{i=1}^{s}$ that are necessary in (3) may be produced by a recursion rule that we extract from the constructive proof of Lemma 2.1.

Let $P_{x, i}$ be the orthogonal projection onto $\operatorname{span}(x) \oplus T_{i}, i=1, \ldots, s$. The base case for the recursion is $q_{1}=P_{x, 1} y$, which is mapped into $x$ by the reflection $\mathrm{h}_{x, q_{1}}$, with the phase factor $z\left(x, q_{1}\right)$. Let the first hop of $y$ towards $x$ be denoted by $\bar{y}_{1}=\mathrm{h}_{x, q_{1}} y$. Assume $y$ has advanced up to $\bar{y}_{k}, k<s$. The recursion rule for $q_{k+1}$ and $\bar{y}_{k+1}$ is the following

$$
q_{k+1}=P_{x, k+1} \bar{y}_{k} \quad \text { and } \quad \bar{y}_{k+1}=\mathrm{h}_{x, q_{k+1}} \bar{y}_{k} .
$$

Recall that $\|x\|=1$ and notice that $\left\|q_{s}\right\|=1$ (which comes out automatically by unitarity). While sequencing the product of reflections (3) by following (5) all the intermediate phase factors $z\left(x, q_{k}\right)$ are absorbed in the definition of every next vector $q_{k+1}$, except for the last one. The phase factor appearing in (3) is $z=z\left(x, q_{s}\right)=q_{s}^{*} x /\left|q_{s}^{*} x\right|$, whenever $\left|q_{s}^{*} x\right|>0$. Otherwise, $z=1$.

Next, we cope the problem of reproducing the evolution operator $U:\left(x_{i} \mapsto y_{i}\right)_{i=0}^{N-1}$ as a succession of Householder reflections. As a preamble, we have to prepare Lemma 2.1 in a special way.

First, the list of subspaces $T_{1}, \ldots, T_{s}$ that had been selected in Lemma 2.1 for the orthogonal direct sum decomposition of the vector space $V=\operatorname{span}(x) \bigoplus_{i} T_{i}$ is denoted by $V^{x}=\left(T_{1}^{x}, \ldots, T_{s}^{x}\right)$ and we write $V=\operatorname{span}(x) \bigoplus V^{x}$. We are explicitly showing $x$ because 
it will not be a fixed vector. In the following, vector $x$ will be each of the basis vectors $\left(x_{0}, \ldots, x_{i} \ldots, x_{N-1}\right)$, one at a time. When the turn of vector $x_{i}$ comes, the relevant vector space $V$ has been contracted to $V_{i}:=\operatorname{span}\left(x_{i}, \ldots, x_{N-1}\right)$ and we must provide a list $V_{i}^{x_{i}} \equiv\left(T_{1}^{x_{i}}, \ldots, T_{s_{i}}^{x_{i}}\right)$ of orthogonal subspaces, such that $V_{i}=\operatorname{span}\left(x_{i}\right) \bigoplus V_{i}^{x_{i}}$ is an orthogonal direct sum decomposition of subspace $V_{i}$. The length $s_{i}>0$ of the list $V_{i}^{x_{i}}$ is at $\operatorname{most} \operatorname{dim}\left(V_{i+1}\right)$, which is attained when all the subspaces in the list are one dimensional.

Second, the phase factor $z$ in the product of the $s$ Householder reflections in $(3)$ is handled in the form

$$
\left[V^{x}\right]_{y}^{*}:=\hat{z} \mathrm{~h}_{x, q_{s}} \cdots \mathrm{h}_{x, q_{1}}
$$

where $\hat{z}=(z-1) P_{x}+\mathbb{I}$, such that it does not affect vectors in $\operatorname{span}(x)^{\perp}$. Again, we do it this way because $x$ will be taking every value in the list $\left(x_{i}\right)_{i=0}^{N-1}$.

The Householder representation theorem of our interest must provide us a way to exchange every pair of basis vectors $x_{i} \leftrightarrow y_{i}$ by following a certain sequence of intermediate vectors.

Proposition 2.1 (Householder representation). Let $X=\left(x_{i}\right)_{i=0}^{N-1}$ and $Y=\left(y_{i}\right)_{i=0}^{N-1}$ be two orthonormal bases of $V$. Let the transformation $U:\left(x_{i} \mapsto y_{i}\right)_{i=0}^{N-1}$. For each $i \in$ $\{1, \ldots, N-2\}$ let $V_{i}$ and $V_{i}^{x_{i}}$ be as described above. Then, there exist vectors $p_{i} \in V_{i}$ such that

$$
U=\left[V_{0}^{x_{0}}\right]_{y_{0}}\left[V_{1}^{x_{1}}\right]_{p_{1}} \cdots\left[V_{N-2}^{x_{N-2}}\right]_{p_{N-2}} \hat{z}
$$

where $\hat{z}=(z-1) P_{x_{N-1}}+\mathbb{I}$ and $z$ is a phase factor.

Proof. Let us start with the $x_{0} \leftrightarrow y_{0}$ exchange. Lemma 2.1 associates to the list of orthogonal subspaces $V_{0}^{x_{0}}$ and to the vector $y_{0}$ the block of reflections $\left[V_{0}^{x_{0}}\right]_{y_{0}}^{*}$ that takes $y_{0}$ into $x_{0}$. Then, the $Y$ basis is transformed into the new orthonormal basis

$$
\left[V_{0}^{x_{0}}\right]_{y_{0}}^{*} Y=\left(x_{0}, y_{1}^{(1)}, y_{2}^{(1)}, \ldots, y_{N-1}^{(1)}\right)
$$

where $y_{j}^{(1)}:=\left[V_{0}^{x_{0}}\right]_{y_{0}}^{*} y_{j}, j>0 . \operatorname{Since} \operatorname{span}\left(y_{1}^{(1)}, \ldots, y_{N-1}^{(1)}\right)=\operatorname{span}\left(x_{1}, \ldots, x_{N-1}\right)=V_{1}$, then by Lemma 2.1 the vector $p_{1}:=y_{1}^{(1)} \equiv\left[V_{0}^{x_{0}}\right]_{y_{0}}^{*} y_{1}$ is taken into $x_{1}$ by the transformation $\left[V_{1}^{x_{1}}\right]_{p_{1}}^{*}$, without touching $x_{0}$ because $x_{0} \perp V_{1}$, we have that $\left[V_{1}^{x_{1}}\right]_{p_{1}}^{*} x_{0}=x_{0}$. The $Y$ basis 
is then transformed into

$$
\left[V_{1}^{x_{1}}\right]_{p_{1}}^{*}\left[V_{0}^{x_{0}}\right]_{y_{0}}^{*} Y=\left(x_{0}, x_{1}, y_{2}^{(2)}, \ldots, y_{N-1}^{(2)}\right)
$$

where $y_{j}^{(2)}:=\left[V_{1}^{x_{1}}\right]_{p_{1}}^{*} y_{j}^{(1)}, j>1$. Again, we have that $\operatorname{span}\left(y_{2}^{(2)}, \ldots, y_{N-1}^{(2)}\right)=V_{2}$ such that the transformation $\left[V_{2}^{x_{2}}\right]_{p_{2}}^{*}$, with $p_{2}:=\left[V_{1}^{x_{1}}\right]_{p_{1}}^{*}\left[V_{0}^{x_{0}}\right]_{y_{0}}^{*} y_{2}$, is a product of reflections that are orthogonal to $\operatorname{span}\left(x_{0}, x_{1}\right)$ (so, it is not touched by the transformation) and so on, as to have the vectors

$$
p_{i}:=\left[V_{i-1}^{x_{i-1}}\right]_{p_{i-1}}^{*} \cdots\left[V_{1}^{x_{1}}\right]_{p_{1}}^{*}\left[V_{0}^{x_{0}}\right]_{y_{0}}^{*} y_{i}
$$

from $i=1$ up to $i=N-2$. At this point, the $Y$ basis has been transformed into the $X$ basis by the following product of blocks of reflections,

$$
U^{*}=\hat{z}^{*}\left[V_{N-2}^{x_{N-2}}\right]_{p_{N-2}}^{*} \cdots\left[V_{1}^{x_{1}}\right]_{p_{1}}^{*}\left[V_{0}^{x_{0}}\right]_{y_{0}}^{*}
$$

where the phase factor $\hat{z}^{*}$ is necessary because unitarity leaves the phase of the (automatic) map $y_{N-1} \mapsto x_{N-1}$ unfixed.

In Proposition 2.1 the lists of orthogonal subspaces, $V_{i}^{x_{i}}$, are quite arbitrary. The only restriction on them is that $\operatorname{span}\left(x_{i}\right) \bigoplus V_{i}^{x_{i}}$ should be an orthogonal direct sum decomposition of $V_{i}$. In the interest of getting the simplest quantum gates we will choose one-dimensional subspaces of $V_{i+1}$ as elements of the list $V_{i}^{x_{i}}$ (this is certainly not the only choice). Neglecting for the moment a permutation of the $X$ basis vectors spanning $V_{i+1}$, we take $T_{k}^{x_{i}}=\operatorname{span}\left(x_{i+k}\right), i=1, \ldots, s_{i}$ such that $V_{i}^{x_{i}}=\left(\operatorname{span}\left(x_{i+k}\right)\right)_{i=1}^{s_{i}}$ and $s_{i}=N-i-1$.

The sequence of reflections $\mathrm{h}_{x_{i}, q_{i, k}}$ conforming the block $\left[V_{i}^{x_{i}}\right]_{p_{i}}$, appearing in (7), is computed using the sequence of $q$-vectors obtained from the recursion in (5), which starts from the vector $p_{i}$ given in (9). For our choice of one-dimensional subspaces in the list $V_{i}^{x_{i}}$, according to Lemma 2.1, every vector $q_{i, k}, k=1, \ldots, s_{i}$, lies in the 2-dimensional $\operatorname{subspace} \operatorname{span}\left(x_{i}\right) \oplus T_{k}^{x_{i}}=\operatorname{span}\left(x_{i}, x_{i+k}\right)$ and can be expanded as $q_{i, k}=a x_{i}+b x_{i+k}$ with coefficients such that $|a|^{2}+|b|^{2}=\left\|q_{i, k}\right\|^{2}$ and $a=|a| z\left(x_{i}, q_{i, k}\right)^{*}$. In the $X$ basis the reflection $\mathrm{h}_{x_{i}, q_{i, k}}$, defined in (1), is specified by the following $\mathrm{U}(2)$ reflection, living in the 
$\operatorname{subspace} \operatorname{span}\left(x_{i}, x_{i+k}\right)$,

$$
\begin{aligned}
\mathrm{h}_{x_{i}, q_{i, k}} x_{i} & =\frac{|a|}{\left\|q_{i, k}\right\|} x_{i}+\frac{z b}{\left\|q_{i, k}\right\|} x_{i+k}, \\
\mathrm{~h}_{x_{i}, q_{i, k}} x_{i+k} & =\frac{z^{*} b^{*}}{\left\|q_{i, k}\right\|} x_{i}-\frac{|a|}{\left\|q_{i, k}\right\|} x_{i+k}, \\
\mathrm{~h}_{x_{i}, q_{i, k}} x_{j} & =x_{j}, \quad \text { whenever } j \neq i, i+k,
\end{aligned}
$$

where $z=z\left(x_{i}, q_{i, k}\right)=a^{*} /|a|, a=x_{i}{ }^{*} q_{i, k}$ and $b=x_{i+k}{ }^{*} q_{i, k}$.

\section{The Householder SCHEME FOR $n$-QUBIT BASES}

The general mathematical scheme we have expounded in the previous section is next adapted to the tensor product character of $n$-qubit states. We proceed in two steps. First, in this Section, we reformulate the hypotheses of Proposition 2.1 in the form of a factoring scheme in the standard (input and target) qubit bases for an array of $n$ qubits. A rich combinatory of factorizations that is inherent to the product character of multi-qubit states is exposed. The second step is to give individual reflections the form of standard quantum gates acting on one-qubit subsystems. The second step is the subject of the next Section.

The standard basis for the $n$-qubit input states is the set of $2^{n}$ product states, $X=$ $\left\{|u\rangle: u \in\{0,1\}^{n}\right\}$, where $|u\rangle \equiv\left|u_{0} u_{1} \cdots u_{n-1}\right\rangle \equiv\left|u_{0}\right\rangle\left|u_{1}\right\rangle \cdots\left|u_{n-1}\right\rangle$. The target $n$ qubit basis $\left.Y:=\{|v\rangle\rangle: v \in\{0,1\}^{n}\right\}$ is to be reached from $X$ by the unitary operation $U \in \mathrm{U}\left(2^{n}\right)$. A double right angle is being used to distinguish the target states $\mid \gg$ from the input states |\rangle . The $2^{2 n}$ amplitudes $\left.\langle u \mid v\rangle\right\rangle$ that are the entries of $U$ are assumed known.

The sequence of blocks of reflections representing $U$ in (7) depend (a) on the order that is given to the product states in the input basis $X$ and (b) on the lists $V_{i}^{u_{i}}$ of onedimensional orthogonal subspaces spanning $V_{i+1}$. In order to fix the ideas, consider the following (a) order for the input basis $X$ of a 3-qubit example

$$
\left(\begin{array}{cccccccc}
|011\rangle, & |001\rangle, & |111\rangle, & |010\rangle, & |100\rangle, & |000\rangle, & |101\rangle, & |110\rangle \\
0, & 1, & 2, & 3, & 4, & 5, & 6, & 7
\end{array}\right) .
$$


and (b) lists of 1-d subspaces,

$$
\begin{aligned}
V_{0}^{011} & =(\langle 010\rangle,\langle 000\rangle,\langle 001\rangle,\langle 101\rangle,\langle 111\rangle,\langle 100\rangle,\langle 110\rangle) \\
V_{1}^{001} & =(\langle 000\rangle,\langle 010\rangle,\langle 101\rangle,\langle 110\rangle,\langle 111\rangle,\langle 100\rangle) \\
V_{2}^{111} & =(\langle 110\rangle,\langle 010\rangle,\langle 100\rangle,\langle 000\rangle,\langle 101\rangle) \\
V_{3}^{010} & =(\langle 101\rangle,\langle 000\rangle,\langle 110\rangle,\langle 100\rangle) \\
V_{4}^{100} & =(\langle 000\rangle,\langle 110\rangle,\langle 101\rangle) \\
V_{5}^{000} & =(\langle 101\rangle,\langle 110\rangle) \\
V_{6}^{101} & =(\langle 110\rangle)
\end{aligned}
$$

such that $V_{i}=\left\langle u_{i}\right\rangle \bigoplus V_{i}^{u_{i}}$, where $\left\langle u_{i}\right\rangle:=\operatorname{span}\left(\left|u_{i}\right\rangle\right)$. Notice that $\left\langle u_{i}\right\rangle$ is excluded from the lists $V_{j}^{u_{j}}$ having $j \geq i$. For instance, $\left\langle u_{0}\right\rangle=\langle 011\rangle$ does not appear in any of the lists in (13). The choices (12) and (13) determine one of the many possible sequences of reflections in (7) and constitutes, in the next Section, a template for the cascade of quantum gates.

The dimension of the state space of $n$-qubits grows exponentially with $n$, as $2^{n}$. This leads to a vast set of options for the Householder representations (7) of $U$, as the following estimate shows us. Subspaces in the list $V_{i}^{u_{i}}$ may be ordered in $\left(2^{n}-1-i\right)$ ! different forms, $i=0$ to $2^{n}-2$, and there are $2^{n}$ ! different orderings of $X$. This places the lower bound for the number of factorizations provided for a given $U$ by Proposition 2.1, see (7), at super-factorial of $2^{n}$, which is $\prod_{j=1}^{2^{n}} j$ !. This vast set of options is not available for single-particle $(n=1)$ Householder representations.

\section{The Householder Quantum gate}

The $\mathrm{U}(2)$ reflections (11) forming the transformation blocks in (7) will be interpreted, when acting on $n$-qubit states, as quantum gates. Let us denote the reflection in (11) by $h(u, v) \equiv \mathrm{h}_{|u\rangle, q}$, where $\operatorname{span}(u, v)$ is the 2 -d subspace that contains the vector $q$ (see the recursion rule (5)). The matrix $h(u, v)$ in the standard basis has the form established in $(11)$,

$$
\begin{aligned}
h(u, v)|u\rangle & =\eta_{3}|u\rangle+\left(\eta_{1}-i \eta_{2}\right)|v\rangle, \\
h(u, v)|v\rangle & =\left(\eta_{1}+i \eta_{2}\right)|u\rangle-\eta_{3}|v\rangle, \\
h(u, v)|w\rangle & =|w\rangle, \quad \text { whenever } w \neq u, v,
\end{aligned}
$$


where $\vec{\eta}:=\left(\eta_{1}, \eta_{2}, \eta_{3}\right)$ is a shorthand for the coefficients in (11), $\vec{\eta}$ is a real vector and $\sum \eta_{i}^{2}=1$. Our aim in this Section is to identify a one-qubit subsystem and a controlled action on it that recreates the transformation (14). By the end of the Section the vector $\vec{\eta}$ is mapped to a quantum gate.

We exploit the tensor-product character of the input basis vectors. One-qubit states that are common to both product states $|u\rangle$ and $|v\rangle$ can be factorized in (14). Denote by $\mathcal{Q}:=\left\{i: u_{i}=v_{i}\right\}$ the subset of qubit indices where the component bits of words $u$ and $v$ coincide. The complement of $\mathcal{Q}$, denoted $\mathcal{Q}^{\prime}$, is not empty: at least one component bit of words $u$ and $v$ have opposite values. A binary word $w$ is decomposed into two shorter words: $w_{\mathcal{Q}}=\left(w_{i}\right)_{i \in \mathcal{Q}}$ and $w_{\mathcal{Q}^{\prime}}=\left(w_{i}\right)_{i \in \mathcal{Q}^{\prime}}$. The decomposition is written $w=\left(w_{\mathcal{Q}}, w_{\mathcal{Q}^{\prime}}\right)$ and $|w\rangle=\left|w_{\mathcal{Q}}\right\rangle\left|w_{\mathcal{Q}^{\prime}}\right\rangle$, correspondingly. By definition we have that $u_{\mathcal{Q}}=v_{\mathcal{Q}}$ and $u_{\mathcal{Q}^{\prime}}=$ $\bar{v}_{\mathcal{Q}^{\prime}}$, where $\bar{v}$ denotes the component-wise binary negation of $v$.

The factor state $\left|u_{\mathcal{Q}}\right\rangle=\left|v_{\mathcal{Q}}\right\rangle$ that is common to $|u\rangle$ and $|v\rangle$ is factorized in (14). Then, the action of $h(u, v)$ on any state $|w\rangle=\left|w_{\mathcal{Q}}\right\rangle\left|w_{\mathcal{Q}}^{\prime}\right\rangle$ leaves the factor state $\left|w_{\mathcal{Q}}\right\rangle$ untouched.

The matrix $h(u, v)$ does not behave as the identity on $|w\rangle$ in (14) only when either $|w\rangle=|u\rangle$ or $|w\rangle=|v\rangle$. The two conditions for this to happen are that $w_{\mathcal{Q}}=u_{\mathcal{Q}} \equiv v_{\mathcal{Q}}$ and that $w_{\mathcal{Q}^{\prime}}$ either is $w_{\mathcal{Q}^{\prime}}=u_{\mathcal{Q}^{\prime}}$ or is $w_{\mathcal{Q}^{\prime}}=v_{\mathcal{Q}^{\prime}}$. Thus, the action of $h(u, v)$ is factorized respect the $\mathcal{Q}$ and $\mathcal{Q}^{\prime}$ groupings of qubits as follows,

$$
h(u, v)|w\rangle=\left|w_{\mathcal{Q}}\right\rangle H\left(\mathcal{Q}^{\prime} \mid u_{\mathcal{Q}}, u_{\mathcal{Q}^{\prime}} \vee v_{\mathcal{Q}^{\prime}}\right)\left|w_{\mathcal{Q}^{\prime}}\right\rangle
$$

where we have introduced the controlled gate

$$
H\left(\mathcal{Q}^{\prime} \mid u_{\mathcal{Q}}, u_{\mathcal{Q}^{\prime}} \vee v_{\mathcal{Q}^{\prime}}\right)\left|w_{\mathcal{Q}^{\prime}}\right\rangle=\left\{\begin{array}{l}
\mathrm{H}\left|w_{\mathcal{Q}^{\prime}}\right\rangle, \quad\left(w_{\mathcal{Q}}=u_{\mathcal{Q}}\right) \wedge\left(\left(w_{\mathcal{Q}^{\prime}}=u_{\mathcal{Q}^{\prime}}\right) \vee\left(w_{\mathcal{Q}^{\prime}}=v_{\mathcal{Q}^{\prime}}\right)\right) . \\
\left|w_{\mathcal{Q}^{\prime}}\right\rangle, \quad \text { otherwise. }
\end{array}\right.
$$

In the argument $\left(\mathcal{Q}^{\prime} \mid u_{\mathcal{Q}}, u_{\mathcal{Q}^{\prime}} \vee v_{\mathcal{Q}^{\prime}}\right)$ of the controlled gate $H, \mathcal{Q}^{\prime}$ denotes the set of target qubits, which are accessed by the transformation $\mathrm{H}$ provided that $w_{\mathcal{Q}}=u_{\mathcal{Q}}$ and that $\left(w_{\mathcal{Q}^{\prime}}=u_{\mathcal{Q}^{\prime}}\right) \vee\left(w_{\mathcal{Q}^{\prime}}=v_{\mathcal{Q}^{\prime}}\right)$. The transformation $\mathrm{H}$ reflects the state $\left|w_{\ell}\right\rangle_{\ell}$ of any qubit $\ell$ selected from the set $\mathcal{Q}^{\prime}$ as to reproduce the coefficients in (14). We proceed to factorize the controlled gate $H(16)$.

Let $\mathcal{Q}_{\ell}^{\prime}:=\mathcal{Q}^{\prime}-\{\ell\}$ and let $F$ be the controlled NOT gate

$$
F\left(\mathcal{Q}_{\ell}^{\prime} \mid 1_{\ell}\right)\left|w_{\mathcal{Q}_{\ell}^{\prime}}\right\rangle=:\left|x_{\mathcal{Q}_{\ell}^{\prime}}\right\rangle= \begin{cases}\left|w_{\mathcal{Q}_{\ell}^{\prime}}\right\rangle, & w_{\ell}=0 \\ \left|\bar{w}_{\mathcal{Q}_{\ell}^{\prime}}\right\rangle, & \text { otherwise }\end{cases}
$$


where $\mathcal{Q}_{\ell}^{\prime}$ is the set of target qubits for $F$, which is controlled by the one-qubit state $\left|w_{\ell}\right\rangle_{\ell}$ such that $x_{\mathcal{Q}_{\ell}^{\prime}}=\left(w_{i}+{ }_{2} w_{\ell}\right)_{i \in \mathcal{Q}_{\ell}^{\prime}}$ (binary addition is being denoted by $\left.+_{2}\right)$. Whenever $w_{\mathcal{Q}^{\prime}}=u_{\mathcal{Q}^{\prime}}$ or $w_{\mathcal{Q}^{\prime}}=v_{\mathcal{Q}^{\prime}}$ the gate $F$ sets the group $\mathcal{Q}_{\ell}^{\prime}$ of qubits in the state $\left|\left(u_{i}+{ }_{2} u_{\ell}\right)_{i \in \mathcal{Q}_{\ell}^{\prime}}\right\rangle$. Then, the binary word $\kappa_{\ell}:=\left(u_{i}+{ }_{2} u_{\ell}\right)_{i \in \mathcal{Q}_{\ell}^{\prime}}$ is the flag to trigger the action of the single-qubit reflection

$$
R\left(\ell \mid u_{\mathcal{Q}}, u_{\mathcal{Q}_{\ell}^{\prime}}\right)\left|w_{\ell}\right\rangle_{\ell}=\left\{\begin{array}{cc}
\eta_{3}|0\rangle_{\ell}+\left(\eta_{1}-i \eta_{2}\right)|1\rangle_{\ell}, & \left(w_{\mathcal{Q}_{\ell}^{\prime}}=\kappa_{\ell}\right) \wedge\left(w_{\ell}=0\right) . \\
\left(\eta_{1}+i \eta_{2}\right)|0\rangle_{\ell}-\eta_{3}|1\rangle_{\ell}, & \left(w_{\mathcal{Q}_{\ell}^{\prime}}=\kappa_{\ell}\right) \wedge\left(w_{\ell}=1\right) . \\
\left|w_{\ell}\right\rangle_{\ell}, & \text { otherwise. }
\end{array}\right.
$$

and the target qubit of $R$ is $\ell \in \mathcal{Q}^{\prime}$.

Lemma 4.1 (Householder gate). Let $h, R$ and $F$ be as defined above. Then,

$$
h(u, v)=F\left(\mathcal{Q}_{\ell}^{\prime} \mid 1_{\ell}\right) R\left(\ell \mid u_{\mathcal{Q}}, u_{\mathcal{Q}_{\ell}^{\prime}}\right) F\left(\mathcal{Q}_{\ell}^{\prime} \mid 1_{\ell}\right)
$$

Proof. Let $|w\rangle=\left|w_{\mathcal{Q}}\right\rangle\left|w_{\mathcal{Q}_{\ell}^{\prime}}\right\rangle\left|w_{\ell}\right\rangle$. The action in operator form of the controlled gate $F$ in $(17)$ is

$$
F\left(\mathcal{Q}_{\ell}^{\prime} \mid 1_{\ell}\right)\left|w_{\mathcal{Q}_{\ell}^{\prime}}\right\rangle\left|w_{\ell}\right\rangle=\left\langle w_{\ell} \mid 0\right\rangle\left|w_{\mathcal{Q}_{\ell}^{\prime}}\right\rangle\left|0_{\ell}\right\rangle+\left\langle w_{\ell} \mid 1\right\rangle\left|\bar{w}_{\mathcal{Q}_{\ell}^{\prime}}\right\rangle\left|1_{\ell}\right\rangle
$$

The action on $|w\rangle$ of the controlled gate $R$ in (18) is

$$
\begin{aligned}
R\left(\ell \mid u_{\mathcal{Q}}, u_{\mathcal{Q}_{\ell}^{\prime}}\right)\left|w_{\mathcal{Q}}\right\rangle\left|w_{\mathcal{Q}_{\ell}^{\prime}}\right\rangle\left|w_{\ell}\right\rangle= & \left(1-\left\langle w_{\mathcal{Q}} \mid u_{\mathcal{Q}}\right\rangle\left\langle w_{\mathcal{Q}_{\ell}^{\prime}} \mid u_{\mathcal{Q}_{\ell}^{\prime}}\right\rangle\right)\left|w_{\mathcal{Q}}\right\rangle\left|w_{\mathcal{Q}_{\ell}^{\prime}}\right\rangle\left|w_{\ell}\right\rangle+ \\
& \left(\left\langle w_{\ell} \mid 0\right\rangle\left(h_{u u}\left|0_{\ell}\right\rangle+h_{u v}\left|1_{\ell}\right\rangle\right)+\left\langle w_{\ell} \mid 1\right\rangle\left(h_{v u}\left|0_{\ell}\right\rangle+h_{v v}\left|1_{\ell}\right\rangle\right)\right) \times \\
& \left\langle w_{\mathcal{Q}} \mid u_{\mathcal{Q}}\right\rangle\left\langle w_{\mathcal{Q}_{\ell}^{\prime}} \mid u_{\mathcal{Q}_{\ell}^{\prime}}\right\rangle\left|u_{\mathcal{Q}}\right\rangle\left|u_{\mathcal{Q}_{\ell}^{\prime}}\right\rangle,
\end{aligned}
$$

Then, a direct calculation shows that

$$
\begin{aligned}
F\left(\mathcal{Q}_{\ell}^{\prime} \mid 1_{\ell}\right) R\left(\ell \mid u_{\mathcal{Q}}, u_{\mathcal{Q}_{\ell}^{\prime}}\right) & F\left(\mathcal{Q}_{\ell}^{\prime} \mid 1_{\ell}\right)|w\rangle= \\
& \left\langle w_{\ell} \mid 0\right\rangle\left(1-\left\langle w_{\mathcal{Q}} \mid u_{\mathcal{Q}}\right\rangle\left\langle w_{\mathcal{Q}_{\ell}^{\prime}} \mid u_{\mathcal{Q}_{\ell}^{\prime}}\right\rangle\right)\left|w_{\mathcal{Q}}\right\rangle\left|w_{\mathcal{Q}_{\ell}^{\prime}}\right\rangle\left|w_{\ell}\right\rangle \\
& +\left\langle w_{\ell} \mid 1\right\rangle\left(1-\left\langle w_{\mathcal{Q}} \mid u_{\mathcal{Q}}\right\rangle\left\langle\bar{w}_{\mathcal{Q}_{\ell}^{\prime}} \mid u_{\mathcal{Q}_{\ell}^{\prime}}\right\rangle\right)\left|w_{\mathcal{Q}}\right\rangle\left|w_{\mathcal{Q}_{\ell}^{\prime}}\right\rangle\left|w_{\ell}\right\rangle \\
& +\left\langle w_{\mathcal{Q}} \mid u_{\mathcal{Q}}\right\rangle\left\langle w_{\mathcal{Q}_{\ell}^{\prime}} \mid u_{\mathcal{Q}_{\ell}^{\prime}}\right\rangle\left\langle w_{\ell} \mid 0\right\rangle\left|w_{\mathcal{Q}}\right\rangle\left(h_{u u}\left|w_{\mathcal{Q}_{\ell}^{\prime}}\right\rangle\left|0_{\ell}\right\rangle+h_{u v}\left|w_{\mathcal{Q}_{\ell}^{\prime}}\right\rangle\left|1_{\ell}\right\rangle\right) \\
& +\left\langle w_{\mathcal{Q}} \mid u_{\mathcal{Q}}\right\rangle\left\langle\bar{w}_{\mathcal{K}_{\ell}^{\prime}} \mid u_{\mathcal{Q}_{\ell}^{\prime}}\right\rangle\left\langle w_{\ell} \mid 1\right\rangle\left|w_{\mathcal{Q}}\right\rangle\left(h_{v u}\left|\bar{w}_{\mathcal{Q}_{\ell}^{\prime}}\right\rangle\left|0_{\ell}\right\rangle+h_{v v}\left|w_{\mathcal{Q}_{\ell}^{\prime}}\right\rangle\left|1_{\ell}\right\rangle\right) .
\end{aligned}
$$

In the cases when either $w=u=\left(u_{\mathcal{Q}}, u_{\mathcal{Q}_{\ell}^{\prime}}, 0_{\ell}\right)$ or $w=v=\left(u_{\mathcal{Q}}, \overline{u_{\mathcal{Q}_{\ell}^{\prime}}}, 1_{\ell}\right)$ we verify that (22) coincides with the first two cases in the definition of $h$ in (14); otherwise (22) does nothing on the vector state $|w\rangle$, as is required in the third line of (14).

The quantum gate (19) that reproduces the action of a Householder reflection $h(u, v)$, is schematically represented [10] in figure 1. The items implicit in Lemma 4.1 that are necessary to attend the subject of next Section are the following. The conditional on the 


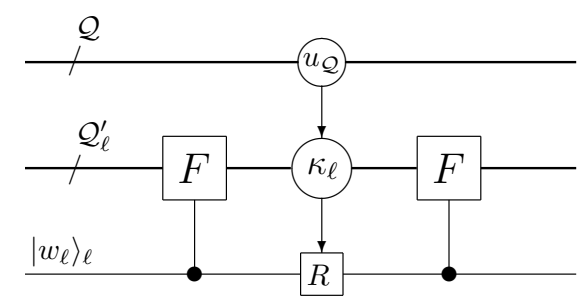

FiguRE 1. A Householder reflection as a quantum gate.

set $\mathcal{Q}$ of coincidences in $(19),\left(w_{\mathcal{Q}}=u_{\mathcal{Q}}\right)$, is tested at the circle labeled $u_{\mathcal{Q}}$, which pulls a trigger if true. A target qubit $\ell$ is selected from the set $\mathcal{Q}^{\prime}$ of differences. Then, the further conditional on the set $\mathcal{Q}_{\ell}^{\prime}$, which is $\left(w_{\mathcal{Q}_{\ell}^{\prime}}=u_{\mathcal{Q}_{\ell}^{\prime}}\right) \vee\left(w_{\mathcal{Q}_{\ell}^{\prime}}=v_{\mathcal{Q}_{\ell}^{\prime}}\right)$, is tested with the help of a CNOT gate $F$ and the circle with the label $\kappa_{\ell}=\left(u_{i}+{ }_{2} u_{\ell}\right)_{i \in \mathcal{Q}_{\ell}^{\prime}}$ in figure 1: if true, another trigger is pulled. Whenever the two triggers are pulled, the $\ell$-th qubit state $\left|w_{\ell}\right\rangle_{\ell}$ is "rotated" by the action of the gate $R_{\ell}=\vec{\eta} \cdot \vec{\tau}$, where $\vec{\tau}=\left(\tau_{1}, \tau_{2}, \tau_{3}\right)$ is the vector of Pauli matrices and the real components of $\vec{\eta}=\left(\eta_{1}, \eta_{2}, \eta_{3}\right)$ are the coefficients of the matrix $h(u, v)$, defined in (18) on the basis of (14) and (11). The full control word to trigger the action of $R_{\ell}$ is $\left(u_{\mathcal{Q}}, \kappa_{\ell}\right)$.

\section{Cascades of Householder gates}

This Section explains how to combine the template described in Section 3 with the H-gate of Lemma 4.1 to assemble cascades of Householder gates that reproduce a given unitary transformation $U$ acting on $n$ qubits. First, we state the formal corollary of Proposition 2.1 and Lemma 4.1 .

Theorem 5.1. An operation on an array of $n$ qubits is unitary if, and only if, the operation is performed by a cascade of Householder gates.

Proof. Householder gates are unitary actions and any cascade assembled with them is unitary too. The converse of this statement was the main subject of previous Sections: Proposition 2.1 establishes that every element of $\mathrm{U}\left(2^{n}\right)$ (considered as a unitary action on a $n$-qubit system) may be represented as the product of reflections of the form given in (11). Then, Lemma 4.1 provides the representation of reflections (11) as (Householder) 


\begin{tabular}{c|cccccc|c}
$k$ & $v_{6}$ & $v_{5}$ & $v_{4}$ & $v_{3}$ & $v_{2}$ & $v_{1}$ & $u$ \\
\hline 0 & 1 & 1 & 1 & 1 & 0 & 0 & 0 \\
1 & 0 & $+1+$ & $+1+$ & 0 & $+1+$ & 0 & 0 \\
2 & $+0+$ & 1 & $+0+$ & 1 & $\emptyset$ & $\emptyset$ & 1 \\
\hline$\ell$ & 0 & 0 & 0 & 0 & 2 & 2 &
\end{tabular}

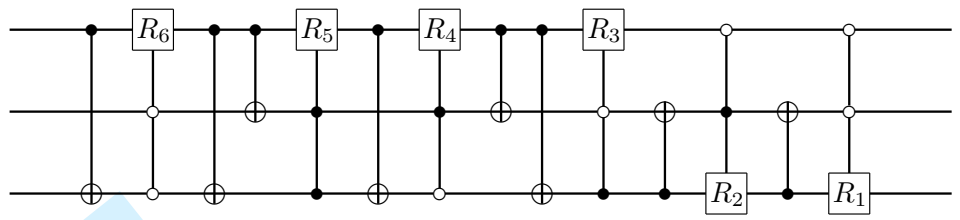

FiguRE 2. The matrix of 1-d subspaces in (23) marked to generate a cascade of Householder gates for the block of reflections $\left[V_{1}^{001}\right]$.

quantum gates, converting the sequence of reflections (7) into a cascade of quantum gates that reproduces any element of $\mathrm{U}\left(2^{n}\right)$.

We proceed to explain how Proposition 2.1 and Lemma 4.1 combine, as pointed out in the proof of Theorem 5.1, to translate every block $\left[V_{i}^{u}\right]_{p_{i}}$ of Householder reflections in the representation (7) of $U$ into a cascade of quantum gates. The assembling steps are illustrated with a 3-qubit example. The starting point is the scheme of Section 3 that establishes the validity of the hypothesis of Proposition 2.1. Let us work out the gate assembling of the block $\left[V_{1}^{u}\right]_{p_{1}}$ of reflections, which has the list of one-dimensional subspaces $V_{1}^{u}=\left(\left\langle v_{i}\right\rangle\right)_{i=1}^{6}$ given in (13). The list $V_{1}^{u}$ directs the reflection-by-reflection transformation of the input state $|u\rangle$, with binary word $u=001$. From the list $V_{1}^{u}$ in (13) we construct the $3 \times 6$ matrix

$$
\mathrm{V}(u)=\left(v_{6}, \ldots, v_{1}\right)=\left(\begin{array}{llllll}
1 & 1 & 1 & 1 & 0 & 0 \\
0 & 1 & 1 & 0 & 1 & 0 \\
0 & 1 & 0 & 1 & 0 & 0
\end{array}\right), \begin{aligned}
& 0 \\
& 0 \\
& 1
\end{aligned}
$$

having as columns the binary words $v_{i}$ in the list $V_{1}^{u}$, entered in reversed order, going from $v_{6}$ down to $v_{1}$, as to fit the order of reflections of the adjoint of the block of reflections in (6). The number of columns, lesser than $2^{n}$, is the number of Householder reflections in the block. Every row of the matrix $\mathrm{V}(u)$ corresponds to a qubit line and every column of $\mathrm{V}(u)$ defines a reflection. We assume the vector $\vec{\eta}$ of every reflection, (14), in every block $\left[V_{1}^{u}\right]_{p_{1}}$ has been computed already, the only task remaining is to convert reflections into the Householder gates in figure 1 . 
Following the description of Lemma 4.1 in the last paragraph of Section 4, at every column of $\mathrm{V}(u)$ the binary words $u$ and $v_{i}$ split the set of qubit lines into the group of coincidences $\mathcal{Q}_{i} \equiv\left\{j: v_{i}(j)=u(j)\right\}$ and the group of differences $\mathcal{Q}_{i}^{\prime} \equiv\left\{j: v_{i}(j) \neq u(j)\right\}$. The extra column on the right side of (23) is word $u=001$, written down as an aid for the comparison of $u$ with individual column words $v_{i}$ of $\mathrm{V}(u)$ and then identify the sets $\mathcal{Q}_{i}$ and $\mathcal{Q}_{i}^{\prime}$. At every column $v_{i}$ the target qubit of the controlled single-qubit rotation $R_{i}$ is any $\ell_{i}$ selected from $\mathcal{Q}_{i}^{\prime}$. Arbitrary choices of $\ell_{i}$ are shown in the matrix $\mathrm{V}(u)$, reproduced in figure 2 , by slashing the corresponding bit value $v_{i}\left(\ell_{i}\right)$. The target qubit $\ell$ is recorded along the bottom line of the table as well. Notice that the target lines $\ell_{i}$ selected in figure 2 have $v_{i}\left(\ell_{i}\right) \neq u\left(\ell_{i}\right)$, as required. The control word for the one-qubit gate $R_{i}$ is $\left(u_{\mathcal{Q}}, \kappa_{\ell}\right)$, where $\kappa_{\ell}=\left(u_{i}+{ }_{2} u_{\ell}\right)_{i \in \mathcal{Q}_{\ell}^{\prime}}$. The two CNOT $F_{i}$ gates that accompany $R_{i}$ in (19), one on each side of the $R$ box in figure 1 , act on the qubit lines in the reduced set of differences $\mathcal{Q}_{\ell_{i}}^{\prime}$. The qubits in $\mathcal{Q}_{\ell_{i}}^{\prime}$ are recognized in the matrix $\mathrm{V}(u)$ in figure $(2)$ by placing a ' + ' sign on each side of the value $v_{i}(j) \neq u(j), j \neq \ell_{i}$. The CNOT gates $F_{i}$ are controlled by the qubit line $\ell_{i}$.

The marking of the matrix $\mathrm{V}(u)(23)$ we have just described, and completed in figure 2, translates directly to a cascade of Householder gates, which is depicted in the schematics below the matrix in figure 2. The single-qubit gates $R_{i}$ are shown as boxes, which are triggered by lines that derive from their control words. A consecutive pair of identical CNOT gates between $R_{4}$ and $R_{5}$ were eliminated.

\section{Minimizing THE NUMBER OF CNOT GATES}

The observation is that a pair of consecutive identical CNOT gates annihilate eachother's action and may be discarded from a cascade of gates. Then, the number of CNOT gates in a cascade of Householder gates is reduced to a minimum by choosing the lists of one-dimensional spaces $V_{i}^{u_{i}}$ in Proposition 2.1 such that the binary words $u_{i}$ follow a Gray order $[11,12]$ where each word differs from the one preceding it in just one bit. We present in the following, without a proof, an algorithm to produce Gray lists of $n$-bit words, in matrix form, that start from a given reference word $u$. 
Assume $u=\left(u_{0}, \ldots, u_{n-1}\right)$ is the given reference word. Then, the algorithm is to produce Gray sequences in the form of a $n \times 2^{n}$ matrix, similar to the table in figure 2, beginning with word $u$. Such a matrix $G(u)$ has a line $G^{i}=\left(g_{0}^{i}, \ldots, g_{2^{n}-1}^{i}\right) \in\{0,1\}^{2^{n}}$ per component bit, $i=0, \ldots, n-1$, with $g_{0}^{i}=u_{i}$. In the algorithm, the relation between the order of production of a line and the place of the line in the matrix $G(u)$ is established through a permutation $i \mapsto k_{i}$ of $\mathbb{Z}_{n}=\{0,1, \ldots, n-1\}$. Every permutation $i \mapsto k_{i}$ generates a different Gray matrix for the reference word $u$. The $2^{n}$ columns of matrix $G(u)$ are all the $n$-bit words presented in Gray's order.

Algorithm 6.1 (Gray matrix). Let the length of binary words be $n>1$ and let the word $u=\left(u_{1}, \ldots, u_{n}\right) \in\{0,1\}^{n}$ be given. Let $i \mapsto k_{i}$ be a permutation of $\mathbb{Z}_{n}$. Then, for every $i \in\{0, \ldots n-2\}$, prepare the binary word

$$
b:=(\underbrace{u_{k_{i}}, \ldots, u_{k_{i}}}_{2^{i} \text { copies }}, \underbrace{\bar{u}_{k_{i}}, \ldots, \bar{u}_{k_{i}}}_{2^{i+1} \text { copies }}, \underbrace{u_{k_{i}}, \ldots, u_{k_{i}}}_{2^{i} \text { copies }})
$$

in order to form the line $G^{k_{i}}$ of the matrix $G(u)$ by concatenating $2^{n-i-2}$ copies of b, $G^{k_{i}}=(\underbrace{b, \ldots, b}_{2^{n-i-2}})$. Matrix $G(u)$ is complete when the last line

$$
G^{k_{n-1}}=(\underbrace{u_{k_{n-1}}, \ldots, u_{k_{n-1}}}_{2^{n-1} \text { copies }}, \underbrace{\bar{u}_{k_{n-1}}, \ldots, \bar{u}_{k_{n-1}}}_{2^{n-1} \text { copies }})
$$

is included. Then, the column words of $G(u)$ follow a Gray order.

Starting with $u=011$ and using the permutation $i \mapsto k_{i}: k_{0}=1, k_{1}=2, k_{2}=0$, Algorithm 6.1 yields the following matrix

$$
G(011)=\left(\begin{array}{llllllll}
0 & 0 & 0 & 0 & 1 & 1 & 1 & 1 \\
1 & 0 & 0 & 1 & 1 & 0 & 0 & 1 \\
1 & 1 & 0 & 0 & 0 & 0 & 1 & 1
\end{array}\right)
$$

Gray sequences presented in the form of matrix $G(u)$ fit nicely to minimize the number of CNOT gates in Householder cascades. For example, the Gray matrix $G(011)$ in (24) may be used to establish the order $\left[V_{0}^{011}\right]\left[V_{1}^{001}\right]\left[V_{2}^{000}\right] \cdots\left[V_{6}^{101}\right]$ of the sequence of blocks in Proposition 2.1 for the 3-qubit example and then apply on the matrices the marking procedure explained in Section 5. The last column word 111 of the Gray matrix in (24) is not necessary and is not used in the 3-qubit example. 
For the first block of reflections, $\left[V_{0}^{011}\right]$, in the sequence we follow the Gray matrix (24) for the word $u=011$. The order of column words in $G(u)$ is reversed first and the rightmost column is taken as $u$ in $\mathrm{V}(u)$. The matrix $\mathrm{V}(011)$ we have derived this way is shown in figure 3-A along with the marking we have chosen. The marking translates into the Householder cascade shown in figure 4-A. Consecutive identical CNOT gates are discarded from the cascade of gates.

For the second block $\left[V_{1}^{001}\right]$ we use a Gray matrix derived from $\mathrm{V}(011)$. We delete the rightmost column word 001 of $\mathrm{V}(011)$ in figure 3-A and take it as the argument of the matrix $\mathrm{V}(001)$, which is shown in figure 3-B. The marking on $\mathrm{V}(001)$ shown corresponds to the cascade of Householder gates in figure 4-B.

The exercise is completed by repeating the procedure on successive blocks of reflections. The matrices so produced, decreasing in length, are shown in figure (3). The corresponding quantum circuits are shown in figure 4 . The seven blocks in figure 4 are assembled together into a single cascade of Householder gates by matching the arrow tails and heads at the ends of the schematics.

\section{FinAl REMARKS}

In the interest of clarity, our presentation did not consider the phase factors $z_{i}$ accompanying each block of reflections in (3). Neither had it considered the late phase factor $z$ in (7). A simple way to incorporate the phase factors into a Householder cascade is to set the global factor to $z=1$ and then individual phase factors $z_{i} z^{*}$ are introduced through the coefficients of the rightmost gate $R$ in the schematics of the $i$-th block, see figure 4 , which are modified as to produce the transformation

$$
\begin{aligned}
& |0\rangle \mapsto\left(z_{i} z^{*}\right) \eta_{3}|0\rangle+\left(z_{i} z^{*}\right)\left(\eta_{1}-i \eta_{2}\right)|1\rangle, \\
& |1\rangle \mapsto\left(\eta_{1}+i \eta_{2}\right)|0\rangle-\eta_{3}|1\rangle .
\end{aligned}
$$

This way to handle the phase factors adds the overall factor $z^{*}$ to the target $Y$-basis while maintaining the original phase differences between vectors in the basis.

The lower bound for the number of factorizations of a unitary action on $n$-qubit systems that are provided by the present implementation of Proposition 2.1 is super-factorial of $2^{n}, \prod_{j=1}^{2^{n}} j$ !. For as few qubits as $n=3$ the number of Householder factorizations has the 




(B)

\begin{tabular}{cccccc|c}
11 & 1 & 1 & 1 & 0 & 0 & 0 \\
$+1+$ & 0 & 0 & 1 & $+1+$ & 0 & 0 \\
1 & 1 & $+0+$ & $+0+$ & $\emptyset$ & $\emptyset$ & 1 \\
\hline \hline
\end{tabular}

(C)

\begin{tabular}{ccccc|c}
\hline \hline 11 & 1 & 1 & 1 & 0 & 0 \\
$+1+$ & 0 & 0 & $+1+$ & $\not$ & 0 \\
$+1+$ & $+1+$ & 0 & 0 & 0 & 0 \\
\hline \hline
\end{tabular}

(D)

\begin{tabular}{cccc|c}
\hline \hline 1 & 1 & 1 & 1 & 0 \\
1 & $+0+$ & $+0+$ & 1 & 1 \\
$+1+$ & $+1+$ & 0 & 0 & 0 \\
\hline \hline
\end{tabular}

(E)

\begin{tabular}{rrr|r}
\hline \hline 1 & 1 & 1 & 1 \\
1 & $\emptyset$ & $\emptyset$ & 1 \\
1 & $+1+$ & 0 & 0 \\
\hline \hline
\end{tabular}

(F)

\begin{tabular}{|c|c|c|c|}
\hline & & & \\
\hline 11 & 1 & 1 & 1 \\
\hline$+1+0$ & 0 & t & 0 \\
\hline 11 & 0 &  & 1 \\
\hline
\end{tabular}

FiguRE 3. Matrices derived from the Gray matrix V(011).

(A)



(B)



(E)

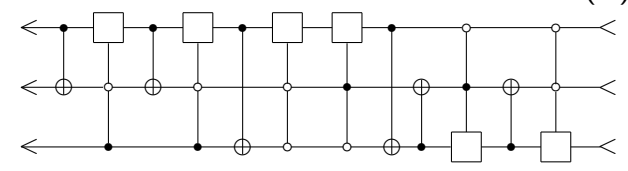

(C)

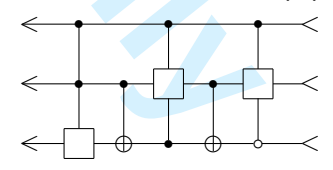

$(G)$
(F)
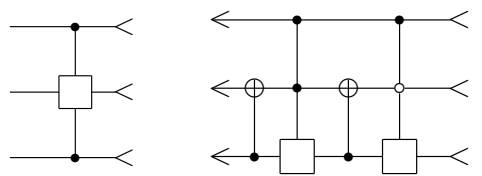

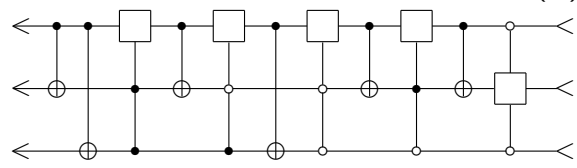

FiguRE 4. Cascades of Householder gates obtained from the matrices in figure 3. 
extremely large lower bound of $\prod_{j=1}^{8}(j !) \approx 5 \times 10^{15}$. The example cascade of Householder gates we have assembled in Section 6 is just one out of a huge set of alternatives.

The assembly method we have developed exploites the Householder representations that are based upon sequences of one-dimensional subspaces in Proposition 2.1. This we did to implement reflections as quantum gates acting on one-qubit subsystems, which are suited to the theoretical requirements of quantum computation. However, quantum simulation in general does not demand such elementary quantum gates. We believe that our method to deal with multi-particle implementations of Householder factorizations may provide some further tools for the design of quantum simulators.

ACKNOWLEDGEMENTS. The author DAQ was supported by CONACyT (Mexico) at the M. of Sc. (Physics) Program of IF-UASLP while the present work was in progress. DAQ is at the University of Leeds, supported by CONACyT (Mexico) too. Our methods were tested using the SciPy tools for Python.

\section{REFERENCES}

[1] D.S. Abrams and S. Lloyd, Simulation of many-body Fermi systems on a universal quantum computer. Phys. Rev. Lett. 79 (1997) 2586.

B. P. Lanyon, et al., Towards quantum chemistry on a quantum computer. Nature Chemistry 2 (2010) 106111.

R. Gerritsma, it et al., Quantum simulation of the Dirac equation. Nature (London) 463 (2010) 68.

K. Kim, it et al., Quantum simulation of frustrated Ising spins with trapped ions. Nature (London) 465 (2010) 590.

B.P. Lanyon, et al., Universal Digital Quantum Simulation with Trapped Ions. Science 334 (2011) 57-61.

J. Struck, et al., Quantum Simulation of Frustrated Classical Magnetism in Triangular Optical Lattices. Science 333 (2011) 996-999.

[2] T. D. Ladd it et al., Quantum computers. Nature 464 (2010) 45-53.

Alán Aspuru-Guzik and Philip Walther, Photonic quantum simulators. Nature Physics 8 (2012) 285291.

Ch. Schneider et al., Experimental quantum simulations of many-body physics with trapped ions. Rep. Prog. Phys. 75 (2012) 024401.

[3] Sadegh Raeisi, et al., Quantum-circuit design for efficient simulation of many-body quantum dynamics. New J. Phys. 14 (2012) 103017. 
Iulia Buluta and Franco Nori, Quantum Simulators; Science 326 (2009) 108-111.

S. Lloyd, Universal quantum simulators; Science 273, 1073 (1996).

[4] J.J. Vartiainen, M. Möttönen and M. M. Salomaa, Efficient Decomposition of Quantum Gates; Phys. Rev. Lett. 92 (2004) 177902.

M. Möttönen, J.J. Vartiainen, V. Bergholm, and M.M. Salomaa, Quantum circuits for general multiqubit gates; Phys. Rev. Lett. 93 (2004) 130502.

Yao-Min Di and Hai-Rui Wei, Synthesis of multivalued quantum logic circuits by elementary gates; Phys. Rev. A 87 (2013) 012325.

[5] Martin Plesch and Caslav Brukner, Quantum-state preparation with universal gate decompositions; Phys. Rev. A 83 (2011) 032302.

[6] Anmer Daskin and Sabre Kais Decomposition of unitary matrices for finding quantum circuits: Application to molecular Hamiltonians J. Chem. Phys. 134 (2011) 144112.

[7] J. Urías, Householder factorizations of unitary matrices. J. Math. Phys. 51 (2010) 072204.

[8] M. Reck, A. Zeilinger, H.J. Bernstein and P. Bertani, Experimental Realization of Any Discrete Unitary Operator. Phys. Rev. Lett. 73 (1994) 58-61.

[9] P. A. Ivanov, E. S. Kyoseva and N. V. Vitanov, Engineering of arbitrary U $(N)$ transformations by quantum Householder reflections. arXiv:0708.2811v1 (2007).

[10] M.A. Nielsen and I.L. Chuang, Quantum Computation and Quantum Information. Cambridge U. Press, 2010.

[11] F. Gray. Pulse code communication, March 17, 1953 (filed Nov. 1947). U.S. Patent 2,632,058.

[12] E. N. Gilbert, Gray codes and paths on the $n$-cube. Bell Syst. Tech. J., 37 (1958) 815-826. 